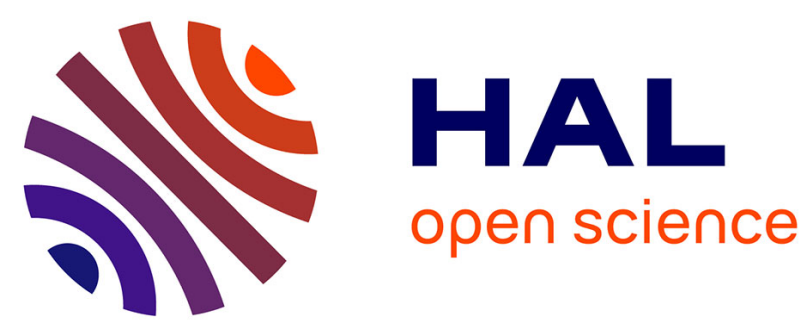

\title{
Spontaneous liquid water dissociation on hybridised boron nitride and graphene atomic layers from ab initio molecular dynamics simulations
}

Benoit Grosjean, Anton Robert, Rodolphe Vuilleumier, Marie-Laure Bocquet

\section{- To cite this version:}

Benoit Grosjean, Anton Robert, Rodolphe Vuilleumier, Marie-Laure Bocquet. Spontaneous liquid water dissociation on hybridised boron nitride and graphene atomic layers from ab initio molecular dynamics simulations. Physical Chemistry Chemical Physics, In press, 10.1039/c9cp06765e . hal$02494473 \mathrm{v} 2$

\section{HAL Id: hal-02494473 \\ https://hal.science/hal-02494473v2}

Submitted on 2 Mar 2020

HAL is a multi-disciplinary open access archive for the deposit and dissemination of scientific research documents, whether they are published or not. The documents may come from teaching and research institutions in France or abroad, or from public or private research centers.
L'archive ouverte pluridisciplinaire HAL, est destinée au dépôt et à la diffusion de documents scientifiques de niveau recherche, publiés ou non, émanant des établissements d'enseignement et de recherche français ou étrangers, des laboratoires publics ou privés. 


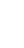

\title{
Spontaneous Liquid Water Dissociation on Hybridised Boron Nitride and Graphene atomic layers from Ab Initio Molecular Dynamics Simulations
}

\author{
Benoît Grosjean, ${ }^{1}$ Anton Robert, ${ }^{1}$ Rodolphe Vuilleumier, ${ }^{1}$ and Marie-Laure Bocquet ${ }^{1, *}$ \\ ${ }^{1}$ PASTEUR, Département de chimie, \\ École normale supérieure, PSL University, \\ Sorbonne Université, CNRS, 75005 Paris, France
}

Abstract

Two-dimensional materials such as graphene $(\mathrm{G})$ and hexagonal boron nitride (BN) have demonstrated potential applications in membrane science and in particular for the harvest of blue energy. Although pure G and BN atomic layers are known to remain inert towards neutral water, one may wonder about the aqueous reactivity of hybridized monolayers formed by joining $\mathrm{BN}$ and $\mathrm{G}$ sheets in a planar fashion. Here, we perform Ab Initio molecular dynamics of liquid water in contact with all possible planar heterostructures. Remarkably, we could observe the spontaneous chemisorption and dissociation of interfacial water molecule into its self-ions, at one specific and non-standard one-dimensional border. Our simulations predict that this type of heterostructure is prone to ionize liquid water already in the absence of any electrical gating.

\footnotetext{
* Correspondence and request for materials should be addressed to M.-L. B. (marie-laure.bocquet@ens.fr)
} 
22 Among the many materials being studied for chemical applications, two-dimensional 23 (2D) ideal materials like graphene (G hereafter) and hexagonal boron nitride (hBN; BN 24 hereafter) are some of the most versatile and interesting ones, thanks to their structural 25 similarity albeit differential properties and especially for BN to its promising applications in 26 blue energy - harvesting energy from salinity gradient in water $[1,2]$. However less is known 27 about how doping in these twin materials alters their interactions with interfacial molecules. 28 Indeed, experimental routes to produce hybrid composites of $\mathrm{BN}$ and $\mathrm{G}$ have been pioneered 29 by $\mathrm{Ci}$ and coworkers [3] and recently summarized in a review [4]. Precisely several routes of 30 synthesis have emerged either from bulk copolymerisation [5] or from growing catalytically 31 on a variety of pure and alloy metal substrates, such as $\mathrm{Cu}$ [6-10], Ru [11-13], Rh [14], Ir 32 [15, 16], Pt[17] or Cu-Ni alloy [18]. The latter fabrication of metal-supported monolayers 33 are being obtained with high levels of control being reported on the nanometer scale thanks 34 to the Scanning Tunneling Microscopy (STM) probe and sometimes real-time Low Energy 35 Electron Microscopy (LEEM). In most of these studies, zigzag (ZZ) linking edges are prefer36 entially formed with respect to the armchair (AC) interface. Moreover in the ZZ edge there 37 is either a C-N interface or a C-B interface - defining donor and acceptor interface states 38 respectively -, the latter being found more favorable [15].

39 Beyond synthesis and characterisation, there is much less progress on the reactivity of the as 40 produced composite border. Using zero Kelvin DFT framework the dissociative reactivity 41 of a gaseous molecules such as di-oxygen $[5,19]$ and water [20] have been explored on a 42 variety of hybridised materials issued from $\mathrm{G}$ and $\mathrm{BN}$. But the reacting molecules were 43 always treated as single frozen species, far from their true environment in the liquid water.

44 Indeed liquid water requires a full quantum treatment to properly include dynamical proton 45 transfers between H-bonding water molecules denoted as the Grotthuss mechanism.[21-23] 46 Closer to the task at hand, M. Sprik has shown in pioneering publications that the full 47 quantum treatment of interfacial water is necessary for correctly describing the proton state 48 at wet surfaces of oxide and clay materials. [24, 25] To our knowledge, a realistic simulation 49 of the interface between water solvent and in-plane BN-G heterostructures remains highly 50 promising albeit challenging. This is the major goal of our study. 
52 From our comparative theoretical study of suspended G and BN monolayers immersed

53 into high pH ionized water [26], both materials are found electrified through the favorable

54 adsorption of the solvated hydroxide ion but in a contrasting manner that gives full support

55 to previous nanofluidics measurements and analysis [1, 27].

56 In this paper we undertake a screening of the possible border configurations when matching

$57 \mathrm{BN}$ and $\mathrm{G}$ monolayer materials in a planar fashion. On all borders we first compute the

58 dissociative adsorption of single water molecule in vacuum and corrected in a dielectric

59 solvent in order to hint at the most favorable ones. On a few selected ones we run extensive

60 Ab Initio Molecular Dynamics (AIMD) with an explicit interface with bulk water. On one

61 border an interfacial $\mathrm{H}_{2} \mathrm{O}$ molecule is found to spontaneously chemisorb onto one boron

62 atom before dissociating by transferring a proton to the surrounding water. These reactive

63 events occur within the few ps of the simulation unveiling the unexpected high reactivity of

64 the hybrid composites.

\section{RESULTS AND DISCUSSION}

\section{Static DFT study}

67 As a start we considered all possible BN-G planar heterojunctions as candidates. The 68 five different junctions and their labelling are displayed in Fig.1, and the corresponding 69 simulation cells are displayed in Supplementary Figure 1. Note that each cell contains two 70 border regions due to periodic boundary conditions. In the interface the border hexagons 71 are necessarily a composite BCN one: for ZZ (AC) border there is an odd (even) number of 72 each material. As a result we have considered the borders ZZ(B) and ZZ(N) and AC that are 73 standard ones. For completeness we have also modelled the BinC and NinC junctions which 74 comprise one single atom of each material. Notably in these junctions the border regions 75 has an extended width of two hexagons while the border region is limited to one hexagon in 76 any of the $\mathrm{ZZ}$ and $\mathrm{AC}$ edge geometries. To our knowledge the BinC and NinC junction have 77 never been characterized experimentally and we denote them non-standard edges. For each 78 junction, several $\mathrm{H}_{2} \mathrm{O}$ dissociative adsorption configurations are possible and inspired by the 79 previous studies $[20,26]$ we restrict them to the ones where i) the hydroxide is chemisorbed 80 on a boron atom, ii) the proton is chemisorbed on a carbon atom, iii) the co-adsorption 
81 occurs within the same hexagon in a cis conformation. The ii) choice is motivated by the 82 exhaustive DFT study of Ref. [20] that shows C-H bonds are significantly stronger than $83 \mathrm{~N}-\mathrm{H}$ bonds when dissociating water on B-doped G and C-doped BN single layers. In total,

8417 adsorption configurations are compared on 5 junction geometries and their dissociative 85 adsorption energies are displayed in Fig. 2. The dissociative adsorption energies of $\mathrm{H}_{2} \mathrm{O}$ 86 called $E_{a d s}$ on different edges were computed first in vacuum and second in implicit water 87 at $T=0 \mathrm{~K}$. To do so, the value of $E_{a d s}$ is obtained by comparing the total energy of the 88 adsorbed ions on the surface $E_{\text {ads/surf }}$ with that of the flat pristine surface $E_{\text {pristine }}$ added 89 to that of the pristine water molecule $E_{\mathrm{H}_{2} \mathrm{O}}$ i.e. $E_{\text {ads }}=E_{\text {ads/surf }}-\left(E_{\text {pristine }}+E_{\mathrm{H}_{2} \mathrm{O}}\right)$. The 90 adsorption energy calculation scheme is illustrated in Supplementary Figure 2.

$91 E_{a d s}$ on pristine atomic layers of BN and $\mathrm{G}$ were also included for comparison and yielded 92 unfavorable dissociative adsorption energies above $2.5 \mathrm{eV}$ (see Supplementary Table 1) in 93 good agreement with previous results [20]. Also our AIMD study of water/BN and water/G 94 interfaces with and without aqueous hydroxide ion gave no indication of water adsorption 95 into these pristine layers. Indeed we showed differential adsorption of aqueous $\mathrm{OH}-$ at 96 these interfaces in quantitative agreement with the measured negative surface charge, hence 97 precluding co-adsorption of protons and consequently excluding water dissociation on the 98 pristine layers. [26] As a general trend already observed in our previous static DFT study 99 [28], the dissociative adsorption energies tend to be destabilized in implicit water versus in 100 vacuum (see the orange triangles in the plot Fig. 2 are mostly lying in the energy scale below 101 the blue circles). In some cases however the energy does not change too much when intro102 ducing a solvent correction. Our energetic results show that the non-standard edges - BinC 103 and NinC on the left side of the plot in Fig. 2 - are more reactive towards water dissociative 104 adsorption than the ZZ edges. AC configurations (right side) are globally the less reactive 105 ones. Precisely, Fig. 2 exhibits three adsorbed configurations with negative (favorable) dis106 sociative adsorption energies in the implicit water case: $\mathrm{BinC} \mathrm{Aa}(-0.88 \mathrm{eV})$ and $\mathrm{BinC} \mathrm{Ba}$ $107(-0.88 \mathrm{eV})$, and NinC Aa $(-0.57 \mathrm{eV})$. These results corroborate the chemical enhancement 108 effect of one-dimensional boundaries in 2D materials. Furthermore, the magnitude of the 109 favourable adsorption energies indicate a strong interaction between the adsorbates and the 110 surface, calling for more realistic dynamical quantum studies on the reactivity of $\mathrm{H}_{2} \mathrm{O}$ at the 111 liquid water / planar heterojunction interface, that we detail below. 
113 Hydrogen bonds are of primordial importance for the water solvent. Therefore implicit 114 solvation schemes can not fully satisfactorily describe the adsorption of $\mathrm{H}_{2} \mathrm{O}$ fragments on $115 \mathrm{BN}$ and $\mathrm{G}$ atomic layers. In fact, the water self-ions $\mathrm{OH}^{-}$and $\mathrm{H}^{+}$resulting from water 116 auto-dissociation are transient species in water because they are subjected to fast proton 117 transfers from H-bonded water molecules. In order to properly simulate such systems, one 118 must resort to computationally expensive $a b$ - initio molecular dynamics (AIMD) simula119 tions in which water molecules are comprehensively described. We therefore computed 10 120 ps long unbiased AIMD trajectories of several adsorption configurations in contact with 195 121 water molecules equilibrated at $323 \mathrm{~K}$ (see Methods). An example of an AIMD simulation 122 cell without adsorbates is displayed in Fig. 3. Due to computational costs only 4 of the 17 123 configurations could be tested by AIMD. The dissociated configurations tested by AIMD 124 correspond to coloured (red and green) labels in Fig. 2: the 3 configurations identified as 125 favourable in implicit water by static DFT (BinC Aa, Ba and BinC Aa) and the most 126 unfavourable dissociation configuration among the ZZ edges: ZZ(N) Ab. This last edge 127 configuration corresponds to the experimentally observed junction geometry. In all 4 cases, 128 the co-adsorbed fragments $\mathrm{OH}^{-}$and $\mathrm{H}^{+}$were found metastable, as none of the adsorbed 129 fragments was desorbing during the $10 \mathrm{ps}$ of the dynamics. More interestingly the most 130 unfavourable of the 4 configurations $(\mathrm{ZZ}(\mathrm{N}) \mathrm{Ab})$ was also found metastable, despite a static 131 adsorption energy computed as high as $+1.97 \mathrm{eV}$. Hence it is likely that all of the 17 config132 urations considered in the present work would be found metastable if tested similarly i.e. by

133 free AIMD simulations. This points out that the theoretical results originating solely from 134 static DFT results might convey a partial picture of the reactivity in water solvent. This 135 stresses even further the necessity of an explicit and quantum description of solvents like 136 water at the interface of $2 \mathrm{D}$ materials.

\section{Spontaneous Reaction}

138 It is crucial to stress that the timescale and the number of chemical objects that one 139 can simulate with AIMD do not allow for the observation of rare chemical events, so that 140 one can only hope to witness extremely easy reactions along an unbiased trajectory. The 
141 observation of any spontaneous reaction is therefore a strong indication of the extremely 142 high reactivity of those surfaces. To better study the water dissociation mechanism, a water 143 interface with a pristine NinC junction i.e. free of fragments, was thermalized at $323 \mathrm{~K}$ 144 while preventing all water molecules to approach the surface below $2 \AA$. Upon release of the 145 latter constraint, a water molecule starting at a large distance of $2.8 \AA$ from the surface (see 146 Fig. 4a, cyan line) i.e. within the interfacial solvent layer (see the inset of Fig. 4b, water 147 density peak at $3.3 \AA$ ), was found to chemisorb on a boron atom of the junction after $t=1.2$ 148 ps of free MD (see Fig. 4c, inset 2). The water adsorbates then rapidly dissociates into an 149 adsorbed hydroxide and an aqueous hydronium at $t=1.3 \mathrm{ps}$ (see Fig. 4c, inset 3). Although 150 the ions dynamically recombine transiently at certain points of the $10 \mathrm{ps}$ long trajectory, 151 the as created hydronium cation diffuses further away from the first solvation shell of the 152 fixed adsorbed $\mathrm{OH}^{-}$. To identify the dissociation state from the atomic configurations, we 153 associate each hydrogen atom to the closest oxygen to it. The atoms with respectively one 154 and three associated hydrogen are then defined as corresponding to the hydroxide and the 155 hydronium. During the simulation the adsorbate is in a $\mathrm{OH}^{-}$state only $94 \%$ of the time 156 and in a $\mathrm{H}_{2} \mathrm{O}$ state $6 \%$ of the remaining time.

157 The distance from the surface of the oxygen that adsorbs and the distance between the 158 two water self-ions are respectively displayed in cyan and green in Fig. 4a. The chaotic 159 trajectory (green line) of the hydronium ion in water is simply the manifestation of its 160 intrinsic reactivity in water: it will transfer one proton to a neighboring water that will 161 become the hydronium species. This results into large diffusion jumps of the hydronium 162 cation and blinking effects when tracking it in the dynamical simulation (see the whole 10 163 ps trajectory in Supplementary Movie 1).

164 We therefore propose a reaction in three steps, as illustrated in Fig. 4c: diffusion of $\mathrm{H}_{2} \mathrm{O}$ 165 near the junction, chemisorption onto a border boron atom and subsequent but immediate 166 dissociation. The last step indeed is seen to occur only once the adsorbed state reached, not 167 during the approach of $\mathrm{H}_{2} \mathrm{O}$ to the surface.

168 Starting from a different atomic configuration, another trajectory simulating the same 169 reactive interface was computed for 21 ps. The chemisorption was once again observed 170 at $t=2.5$ ps-, corroborating the likeliness of this reaction (see Supplementary Figure 3). 171 Yet, although one proton of the adsorbates appears to be shared with a solvating water 172 molecule, no clear dissociation occurs, i.e. with diffusion of the excess proton further than 
173 the first solvation layer. With the method described above to identify the water self-ions 174 from simulation, the adsorbate is in a $\mathrm{OH}^{-}$state only $15 \%$ of the time and in a $\mathrm{H}_{2} \mathrm{O}$ state $17585 \%$ of the remaining time. Thus by comparing the two trajectories on the same junction 176 starting with different solvent configuration, it appears that the organization of the solvation 177 shell around the water adsorbate is playing a key role for its dissociation. This is expected 178 from the strong role of solvent reorganisation in the proton diffusion process [21, 22] but is 179 magnified here by sequential adsorption and dissociation processes. Further studies would 180 be necessary to better understand how the solvent re-organization is coupled to these two 181 events.

182 Taking into account periodic boundary conditions (PBC) of the simulation, the spontaneous 183 dissociative adsorption of $\mathrm{H}_{2} \mathrm{O}$ occurs at least once every $1.5 \mathrm{~nm}$ which corresponds to 184 roughly $1 / 6$ of the border boron sites being bonded to an oxygen atom. This estimate being 185 based on the observation of the reaction in a limited time - 10 ps long AIMD trajectory-, 186 we expect the actual occurrence to be much higher.

187 In the following, we aim at quantifying the reactivity of $\mathrm{H}_{2} \mathrm{O}$ with the NinC junction 188 by determining the free energy of adsorption of a water molecule. This was derived from 189 the potential of mean force (PMF) obtained by umbrella sampling while moving the adsor190 bate away from the surface to the first interfacial layer of solvent by restraining the oxygen 191 distance of the interacting water to the surface (see Methods). Because of the very high 192 reactivity of the interface, preventing all other water molecules of the simulation cell from 193 approaching the surface at less than $2 \AA$ was proven necessary to avoid spontaneous adsorp194 tion. This is similar to constraining coordination numbers for the computation of pKa's to 195 avoid unwanted re-protonation of the acid.[29, 30] The resulting free energy profile shown in 196 Fig. 4b displays a chemisorption well of - $43 \mathrm{meV}$ located $1.6 \AA$ away from the surface and 197 a barrier of $90 \mathrm{meV}$ at $2 \AA$. The basin around $3 \AA$ corresponds to the water density peak 198 of the first water layer at $3.3 \AA$ from the surface. The minimum of this basin was taken 199 as reference energy for the PMF (see Fig. 4b, horizontal grey dotted line). Interestingly, 200 the barrier height corresponds solely to about $4 k_{B} T$, while a large number of potentially 201 reacting molecules are present close to the junction as $\mathrm{H}_{2} \mathrm{O}$ is the solvent, which furthermore 202 displays a density peak in the vicinity of the surface.

203 How realistic are our predicted reactive junctions towards liquid water? Experimentally 204 the observed ZZ border is mostly the $\mathrm{ZZ}(\mathrm{B})$ one $[8,15]$ and not the one that we found 
205 highly reactive i.e. NinC. Hence we tested a similar reactivity at the ZZ(B) border [8, 15]

206 by thermalizing, i.e. running MD at room temperature, a simulation cell consisting of 207 one $\mathrm{H}_{2} \mathrm{O}$ adsorbed on the border boron atom in plain water by restraining the adsorbed 208 water molecule at $1.6 \AA$ from the surface. Upon release of the latter restraint the water 209 molecule was found to spontaneously desorb within a few hundreds of femtoseconds, as 210 displayed in Supplementary Movie 2. Nevertheless, the adsorption configuration ZZ(B) Ba

211 was also identified as metastable i.e the $\mathrm{OH}$ et $\mathrm{H}$ co-fragments remain stable during free

212 MD (see previous section). Hence we postulate that the metastable adsorption state of the

213 water fragment could be reached from ions already separated in solution or via concerted

214 formation of the B-O bond and rupture of one $\mathrm{O}-\mathrm{H}$ bond.

215 Nevertheless even if sharp 1D interfaces with NinC edge hexagons are not likely to be 216 observed in planar heterojunctions, we infer that these asymmetric hexagons can still be 217 present locally in a zero dimensionality of defects like G-doped BN and vice versa BN-doped 218 graphene materials. Thus we hope that our results can forster such experiments to undertake 219 a controlled reactivity study of environmental molecules on these composite BCN materials.

\section{CONCLUSION}

221 By means of ab initio calculations, we have evidenced a covalent chemistry on one water222 immersed BN-G planar heterostructure, chemistry that does not exist on pristine materials. 223 A large variety of border geometries were tested and the dissociative adsorption of $\mathrm{H}_{2} \mathrm{O}$ to 224 form $\mathrm{B}-\mathrm{OH}^{-}$and $\mathrm{C}-\mathrm{H}^{+}$fragments was found energetically favorable in a few cases. One 225 junction geometry was identified as particularly reactive with spontaneous adsorption of $226 \mathrm{H}_{2} \mathrm{O}$ and subsequent dissociation into an adsorbed hydroxide and an aqueous hydronium.

227 This reactive junction comprises one row of BN hexagons with one $\mathrm{C}$ atom substituting $\mathrm{B}$ 228 and the next adjacent row of $\mathrm{G}$ hexagons with one $\mathrm{N}$ atom substituting $\mathrm{C}$. Moreover such 229 asymmetric composite hexagons echoe substitutional doping in 2D materials and hence may 230 be also observed in patchy BN islands embedded in a graphene sheet and vice versa[5, 31]. 231 The use of state-of-the-art techniques such as atomic force microscopy in water is an exquisite 232 tool of choice to seek experimental evidence for the water adsorption and dissociation at the $233 \mathrm{hBN}$-graphene junction. This unique tool requires an ultimate reduction of the noise and has 234 been developed recently $[32,33]$. We hope that our prediction will trigger more reactivity 
studies on chemically doped 2D materials.

\section{METHODS}

237 Both DFT and AIMD simulations were performed using orthorhombic cells containing a 238 hexagonal sheet consisting of 72 Carbon, 36 Boron and 36 Nitrogen atoms. To model the 239 junctions of the zigzag and armchair type, $14.77 \AA \times 25.58 \AA$ and $12.78 \AA \times 29.55 \AA$ single

240 layers were respectively used, separated by $15 \AA$ of vacuum for static DFT calculations and $24121 \AA$ of explicit water for AIMD trajectories. The top views of the neutral Lewis structures 242 of the three investigated cells are displayed in Supplementary Fig. 1a-c. The first and the 243 second cell parameters are respectively parallel to the hybridised junction and perpendicular 244 to the surface. The hBN and graphene primitive lattice parameters $\boldsymbol{a}(2.51 \AA$ and $2.47 \AA$ 245 respectively) evaluated within the present DFT framework exhibit a mismatch of $0.04 \AA$, in 246 agreement with previous calculations using a similar method[28, 34]. The lattice parameter 247 of hBN was chosen to construct the cell, therefore inducing strain in the counterpart network. 248 Because of the lattice mismatch, only a large supercell containing thousands surface atoms 249 could prevent inducing strain in the layer. Static DFT calculations were performed using 250 the Vienna Ab Initio Simulation Package (VASP) in its version 5.4.1, with a $800 \mathrm{eV}$ cutoff 251 of the plane-wave expansion of the wave functions. The electronic cores were described 252 by pseudo-potentials under the projector augmented wave method [35]. The framework of 253 the generalized gradient approximation (GGA) was used to evaluate exchange-correlation 254 energies, with the so-called optPBE functional developed by Klimeš, Bowler and Michaelides 255 [36]. The first Brillouin zone of the supercell was sampled at the gamma point. Solvation 256 energies in implicit water were evaluated within the approach implemented into VASP by 257 Mathew and Hennig [37]. In this scheme, the solvent is represented as a dielectric constant 258 which is a functional of the electronic density, continuously spanning values from 0 to 80 , 259 the bulk water value asymptotically approached in regions where the electronic density is 260 lower than $\rho_{\text {cut }}=0.0025 \AA^{3}$. All atomic configurations which energy was used to derive 261 adsorption strengths were obtained after relaxing the structure to reach atomic forces below $2620.05 \mathrm{eV} / \AA$. A typical VASP input for a static DFT calculation with implicit water is shown 263 in Supplementary Note 1.

264 AIMD was performed with the CP2K 5.1 code[38]. The forces were computed using density 
265 functional theory (DFT) as implemented in the QuickStep module[39, 40]. Wavefunctions 266 were projected over DZVP-MOLOPT-SR-GTH basis sets [41] and planewaves expanded to 267 a 600 Ry energy cutoff. Geodecker-Teter-Hutter pseudopotentials were used to represent 268 electronic cores [42-44]. The Perdew, Burke and Ernzerhof (PBE) functional was used[45] 269 with the D3 dispersion correction scheme[46, 47]. In order to limit nuclear quantum effects 270 and to reduce the time step size needed for energy conservation in our Born-Oppenheimer 271 AIMD, the mass of all protons was substituted by that of deuterium. A time step of 0.5 272 fs was used for the dynamics. AIMD simulations were carried out in the NVT ensemble 273 at $323 \mathrm{~K}$ using Nose-Hoover thermostats $[48,49]$ with a time constant of $500 \mathrm{fs}$. The PBE 274 functional predicts a reasonable liquid water structure at this slightly increased temperature 275 condition. The $21 \AA$ high simulation cell contained $195 \mathrm{H}_{2} \mathrm{O}$, yielding a pressure $\langle P\rangle=2 \pm$ $2763 \mathrm{MPa}$ (see Supplementary Methods), and was built as follows. Using the Packmol software 277 [50], the water molecules were randomly placed inside a $16 \AA$ high cell which was later 278 juxtaposed to the surface with a $2.5 \AA$ vacuum separation between the hexagonal layer and 279 the liquid. The system was heated up to $600 \mathrm{~K}$ before cooling down to $323.15 \mathrm{~K}$, followed by 280 a 3 ps equilibration. To test the metastability of the adsorbed state the two adsorbates were 281 added to the simulation cell and restrained close to the surface for 2.5 ps before removal of 282 the biases.

283 To obtain the potential of mean force of a water molecule close to the junction, the distance 284 of one particular water oxygen to the surface was restrained using a harmonic bias with a 285 force constant of $0.1 \mathrm{Ha} /$ Bohr. This collective variable was defined as the oxygen distance 286 to the closest surface atom. The target of the restraint was progressively moved from $2871.4 \AA$ to $3.6 \AA$ along successive trajectories of 5 ps which starting point corresponds to 2880.05 to $1 \mathrm{ps}$ of the previous run. The two oxygen-hydrogen bond of the target $\mathrm{H}_{2} \mathrm{O}$ were 289 restrained by a harmonic potential centered at $1 \AA$ with a force constant of $0.025 \mathrm{Ha} / \mathrm{Bohr}$. 290 Simultaneously, harmonic biases with a force constant of $0.1 \mathrm{Ha} /$ Bohr were applied to each 291 combination of another oxygen atom and any boron atom. The restraints were only applied 292 when the oxygen-boron distance would come close to $2 \AA$ in order to prevent spontaneous 293 adsorptions. To define the collective variables and apply the biases, the open-source and 294 community developed PLUMED library[51] was used in its version 2.4[52] as implemented 295 in the CP2K 5.1 code. Typical CP2K and PLUMED input files used for biased dynamics 296 can be respectively found in Supplementary Note 2 and Supplementary Note 3. Further 
297 details regarding the PLUMED bias can be found in Supplementary Methods. To derive 298 the free energy profile, the biased trajectories were analyzed using a home implementation 299 of the Weighted Histogram Analysis Method (WHAM) developed by Kumar et al. [53] as

300 formulated by Souailles and Roux in their extension of this protocol[54]. A bin size of $0.05 \AA$ 301 was choose to compute the histograms. Evidence of convergence of the WHAM analysis used 302 to derive the PMF of Fig. 4b is displayed in Supplementary Figure 4. The final snapshot of 303 the trajectory with the $\mathrm{H}_{2} \mathrm{O}$ restrained at 3.2 (3.0) $\AA$ from the surface was used as starting 304 point for the bias-free trajectory leading to spontaneous adsorption of a water molecule, 305 with (without) further dissociation. The water density profile displayed in inset in Fig. 4b 306 was evaluated over $30 \mathrm{ps}$ of trajectory corresponding to the gathering of the two simulations 307 of the NinC junction in water. More detailed profiles are displayed in Supplementary Figure 3085.

\section{REFERENCES}

310 [1] Siria, A. et al. Giant osmotic energy conversion measured in a single transmembrane boron 311 nitride nanotube. Nature 494, 455-458 (2013).

312 [2] Siria, A., Bocquet, M.-L. \& Bocquet, L. New avenues for the large-scale harvesting of blue energy. Nat. Rev. Chem. 1, 0091 (2017).

[3] Ci, L. et al. Atomic layers of hybridized boron nitride and graphene domains. Nat. Mater. 9, 430-435 (2010).

[4] Hus, S. M. \& Li, A.-P. Spatially-resolved studies on the role of defects and boundaries in electronic behavior of 2D materials. Prog. Surf. Sci. 92, 176-201 (2017).

[5] Chen, S. et al. Designing boron nitride islands in carbon materials for efficient electrochemical synthesis of hydrogen peroxide. J. Am. Chem. Soc. 140, 7851-7859 (2018).

[6] Levendorf, M. P. et al. Graphene and boron nitride lateral heterostructures for atomically thin circuitry. Nature 488, 627-632 (2012).

[7] Kim, S. M. et al. Synthesis of patched or stacked graphene and hBN flakes: A route to hybrid structure discovery. Nano Lett. 13, 933-941 (2013).

[8] Han, G. H. et al. Continuous growth of hexagonal graphene and boron nitride in-plane heterostructures by atmospheric pressure chemical vapor deposition. ACS Nano 7, 10129-10138 
(2013).

[9] Liu, L. et al. Heteroepitaxial growth of two-dimensional hexagonal boron nitride templated by graphene edges. Science 343, 163-167 (2014). URL https://doi.org/10.1126\%2Fscience. 1246137.

[10] Zhuang, P. et al. Growth of lateral graphene/h-BN heterostructure on copper foils by chemical vapor deposition. Nanotechnology 30, 03LT01 (2018).

[11] Sutter, P., Cortes, R., Lahiri, J. \& Sutter, E. Interface formation in monolayer graphene-boron nitride heterostructures. Nano Lett. 12, 4869-4874 (2012).

[12] Sutter, P., Huang, Y. \& Sutter, E. Nanoscale integration of two-dimensional materials by lateral heteroepitaxy. Nano Lett. 14, 4846-4851 (2014).

[13] Liu, M. et al. Quasi-freestanding monolayer heterostructure of graphene and hexagonal boron nitride on ir(111) with a zigzag boundary. Nano Lett. 14, 6342-6347 (2014).

[14] Gao, Y. et al. Toward single-layer uniform hexagonal boron nitride-graphene patchworks with zigzag linking edges. Nano Lett. 13, 3439-3443 (2013).

[15] Drost, R. et al. Electronic states at the graphene-hexagonal boron nitride zigzag interface. Nano Lett. 14, 5128-5132 (2014).

[16] Petrović, M., von Hoegen, M. H. \& zu Heringdorf, F.-J. M. Lateral heterostructures of hexagonal boron nitride and graphene: BCN alloy formation and microstructuring mechanism. Appl. Surf. Sci. 455, 1086-1094 (2018).

[17] Nguyen, T. H. et al. Microscopic insight into the single step growth of in-plane heterostructures between graphene and hexagonal boron nitride. Adv. Mater. Interfaces 12, 675-682 (2019).

[18] Zhang, S., Li, J., Wu, H., Li, X. \& Guo, W. Direct synthesizing in-plane heterostructures of graphene and hexagonal boron nitride in designed pattern. Adv. Mater. Interfaces 5, 1800208 (2018).

[19] Sun, Q., Sun, C., Du, A., Dou, S. \& Li, Z. In-plane graphene/boron-nitride heterostructures as an efficient metal-free electrocatalyst for the oxygen reduction reaction. Nanoscale $\mathbf{8}, 14084$ 14091 (2016).

[20] Al-Hamdani, Y. S., Alfè, D., von Lilienfeld, O. A. \& Michaelides, A. Tuning dissociation using isoelectronically doped graphene and hexagonal boron nitride: Water and other small molecules. J. Chem. Phys. 144, 154706 (2016). 
356 [21] Tuckerman, M., Laasonen, K., Sprik, M. \& Parrinello, M. Ab initio molecular dynamics simulation of the solvation and transport of hydronium and hydroxyl ions in water. $J$. Chem. Phys. 103, 150-161 (1995).

[22] Tuckerman, M., Laasonen, K., Sprik, M. \& Parrinello, M. Ab Initio Molecular Dynamics Simulation of the Solvation and Transport of $\mathrm{H} 3 \mathrm{o}+$ and $\mathrm{OH}-$ Ions in Water. J. Phys. Chem.

362

363

364 99, 5749-5752 (1995).

[23] Chen, M. et al. Hydroxide diffuses slower than hydronium in water because its solvated structure inhibits correlated proton transfer. Nat. Chem. 10, 413-419 (2018).

[24] Cheng, J. \& Sprik, M. Acidity of the Aqueous Rutile TiO2(110) Surface from Density Functional Theory Based Molecular Dynamics. J. Chem. Theory Comput. 6, 880-889 (2010).

[25] Tazi, S., Rotenberg, B., Salanne, M., Sprik, M. \& Sulpizi, M. Absolute acidity of clay edge sites from ab-initio simulations. Geochimica et Cosmochimica Acta 94, 1-11 (2012).

[26] Grosjean, B., Bocquet, M.-L. \& Vuilleumier, R. Versatile electrification of two-dimensional nanomaterials in water. Nat. Comm. 10 (2019).

[27] Secchi, E., Niguès, A., Jubin, L., Siria, A. \& Bocquet, L. Scaling behavior for ionic transport and its fluctuations in individual carbon nanotubes. Phys. Rev. Lett. 116, 154501 (2016).

[28] Grosjean, B. et al. Chemisorption of hydroxide on 2D materials from DFT calculations: Graphene versus hexagonal boron nitride. J. Phys. Chem. Lett. 7, 4695-4700 (2016).

[29] Sprik, M. Coordination numbers as reaction coordinates in constrained molecular dynamics. Faraday Discussions 110, 437-445 (1998).

[30] Sprik, M. Computation of the pK of liquid water using coordination constraints. Chemical Physics 258, 139-150 (2000).

[31] Lu, J. et al. Order-disorder transition in a two-dimensional boron-carbon-nitride alloy. Nat. Comm. 4 (2013).

[32] Schlesinger, I., Kuchuk, K. \& Sivan, U. An ultra-low noise optical head for liquid environment atomic force microscopy. Rev. Sci. Instrum. 86, 083705 (2015).

[33] Kuchuk, K. \& Sivan, U. Hydration structure of a single DNA molecule revealed by frequencymodulation atomic force microscopy. Nano Lett. 18, 2733-2737 (2018).

[34] Graziano, G., Klimeš, J., Fernandez-Alonso, F. \& Michaelides, A. Improved description of soft layered materials with van der waals density functional theory. J. Phys.: Condens. Matter 24, 424216 (2012). 
387 [35] Kresse, G. \& Joubert, D. From ultrasoft pseudopotentials to the projector augmented-wave method. Phys. Rev. B 59, 1758-1775 (1999).

389 [36] Klimeš, J., Bowler, D. R. \& Michaelides, A. Chemical accuracy for the van der waals density functional. J. Phys.: Condens. Matter 22, 022201 (2009).

391 [37] Mathew, K., Sundararaman, R., Letchworth-Weaver, K., Arias, T. A. \& Hennig, R. G. Implicit solvation model for density-functional study of nanocrystal surfaces and reaction pathways. $J$. Chem. Phys. 140, 084106 (2014).

[38] Hutter, J., Iannuzzi, M., Schiffmann, F. \& VandeVondele, J. CP2K: atomistic simulations of condensed matter systems. Wiley Interdiscip. Rev. Comput. Mol. Sci. 4, 15-25 (2013).

[39] VandeVondele, J. et al. Quickstep: Fast and accurate density functional calculations using a mixed Gaussian and plane waves approach. Comput. Phys. Commun. 167, 103-128 (2005).

[40] Lippert, G., Hutter, J. \& Parrinello, M. A hybrid gaussian and plane wave density functional scheme. Mol. Phys. 92, 477-488 (1997).

400 [41] VandeVondele, J. \& Hutter, J. Gaussian basis sets for accurate calculations on molecular systems in gas and condensed phases. J. Chem. Phys. 127, 114105 (2007).

410 [46] Grimme, S., Antony, J., Ehrlich, S. \& Krieg, H. A consistent and accurate ab initio 411 parametrization of density functional dispersion correction (DFT-d) for the 94 elements HPu. J. Chem. Phys. 132, 154104 (2010).

413 [47] Grimme, S., Ehrlich, S. \& Goerigk, L. Effect of the damping function in dispersion corrected density functional theory. J. Comput. Chem. 32, 1456-1465 (2011).

415 [48] Nosé, S. A molecular dynamics method for simulations in the canonical ensemble. Mol. Phys. $416 \quad 52,255-268(1984)$. 
417 [49] Nosé, S. A unified formulation of the constant temperature molecular dynamics methods. $J$. 418 Chem. Phys. 81, 511-519 (1984).

419 [50] Martinez, L., Andrade, R., Birgin, E. G. \& Martinez, J. M. PACKMOL: A package for building 420 initial configurations for molecular dynamics simulations. J. Comput. Chem. 30, 2157-2164 421 (2009).

422 [51] The PLUMED consortium: A community effort to promote openness, transparency and re423 producibility in molecular simulations (Submitted).

424 [52] Tribello, G. A., Bonomi, M., Branduardi, D., Camilloni, C. \& Bussi, G. PLUMED 2: New 425 feathers for an old bird. Comput. Phys. Commun. 185, 604-613 (2014).

426 [53] Kumar, S., Rosenberg, J. M., Bouzida, D., Swendsen, R. H. \& Kollman, P. A. The weighted 427 histogram analysis method for free-energy calculations on biomolecules. J. Comput. Chem. 428 13, 1011-1021 (1992).

429 [54] Souaille, M. \& Roux, B. Extension to the weighted histogram analysis method: combining 430 umbrella sampling with free energy calculations. Comput. Phys. Commun. 135, 40-57 (2001).

\section{$431 \quad$ CONFLICS OF INTEREST}

432 There are no conflicts of interest to declare.

\section{ACKNOWLEDGEMENTS}

434 This work was performed on the French national supercomputer Occigen under the DARI 435 grants A0030807364 and A0030802309. M.L.B acknowlegdes funding from the EU H2020 436 Framework Programme/ERC Advanced Grant agreement number 785911—Shadoks. 


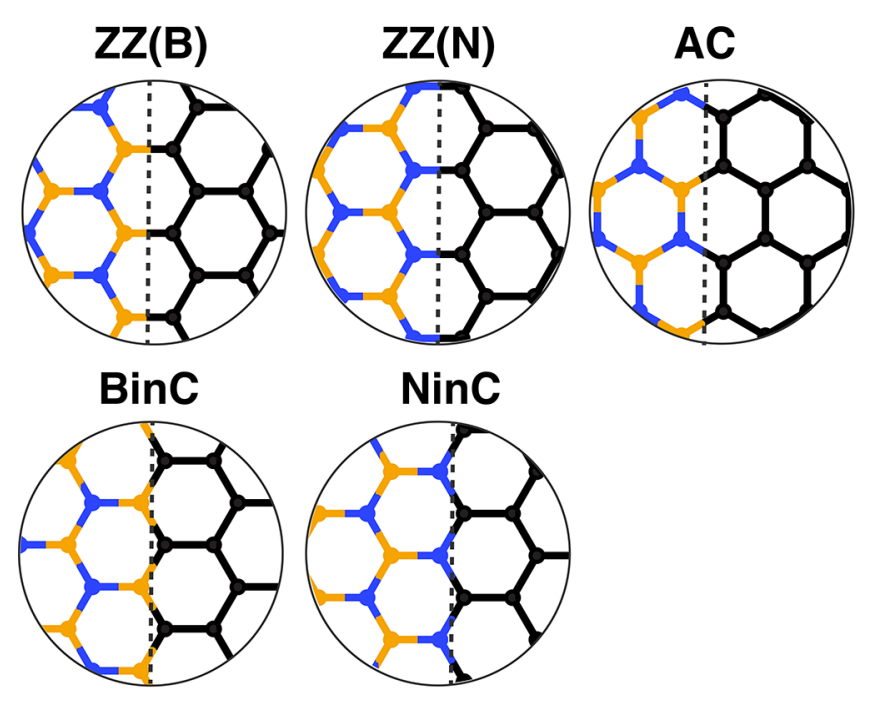

Figure 1. Possible junction geometries for the planar heterostructure and their acronyms. ZZ (AC) stands for zigzag (armchair) edges where the composite hexagons comprises 3 (4 or 2) atoms from BN and 3 (2 or 4) atoms from GR. For the zigzag boundaries, there is either a C-B interfacial bond called ZZ(B) or a C-N bond called $\mathrm{ZZ}(\mathrm{N})$. The junctions BinC and NinC correspond to a composite hexagon formed with one single atom $\mathrm{B}$ and $\mathrm{N}$ respectively from $\mathrm{BN}$ and the rest from GR. The junction line is schematically represented by a black dotted line while the boron, carbon and nitrogen atoms are respectively displayed in orange, black and blue. 


\section{BinC NinC ZZ(B) ZZ(N) AC}

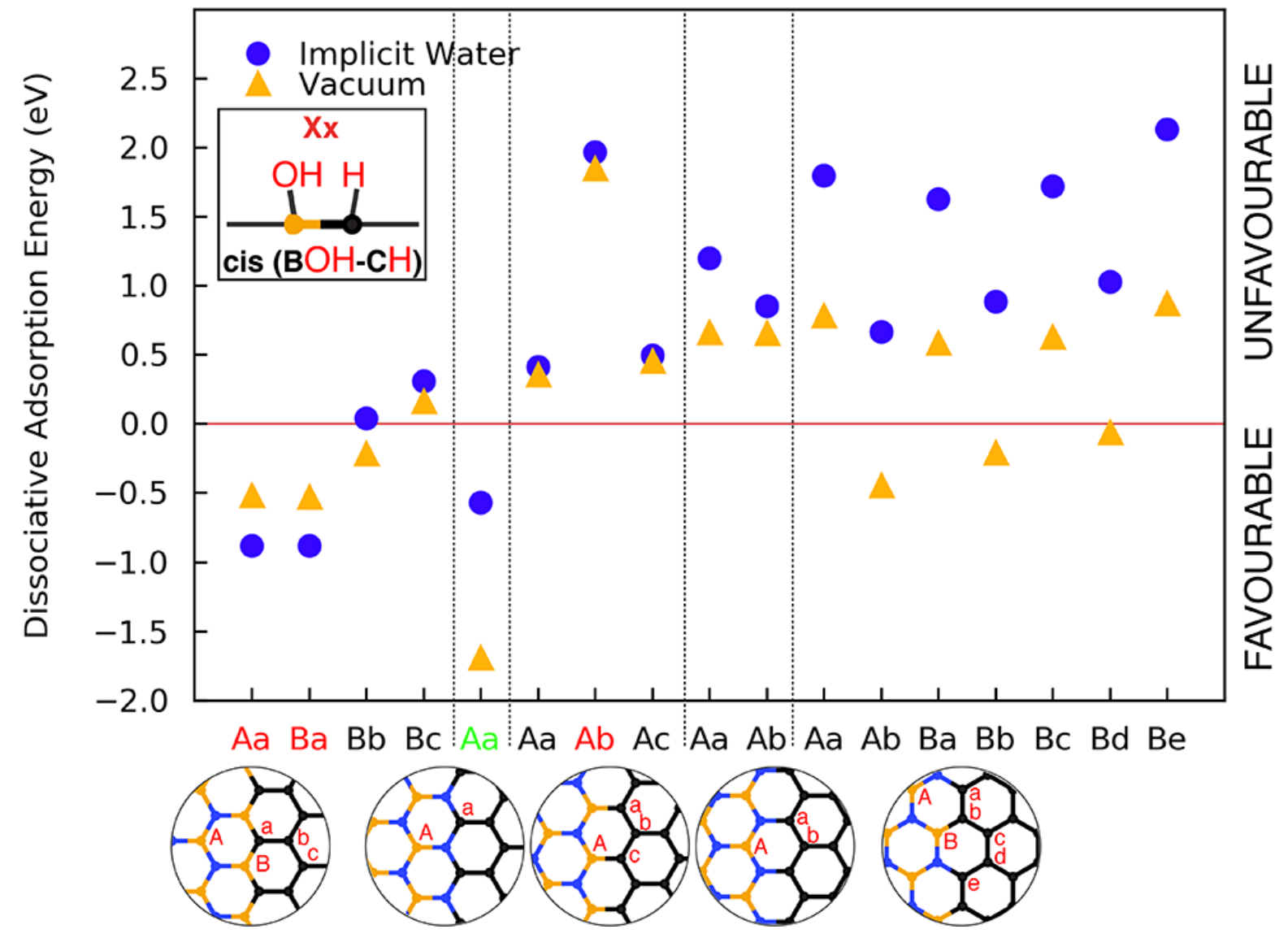

Figure 2. Static DFT $\mathrm{H}_{2} \mathrm{O}$ Dissociative Adsorption Energies limited to $\mathrm{OH}$ fragment bonded to a boron atom while $\mathrm{H}$ fragment bonded to a carbon atom in the same composite hexagon of the heterostructure. Dissociative adsorption energies of $\mathrm{H}_{2} \mathrm{O}$ in vacuum (orange triangles) and implicit water (blue circles) for various adsorption configurations on 5 distinct junction geometries defined in Fig. 1. Red uppercase letters label hydroxide adsorption sites while red lowercase letters indicate associated proton adsorption sites. Coloured labels indicate configuration which meta-stability was also tested in explicit water by ab-initio molecular dynamics. Spontaneous dissociative adsorption of $\mathrm{H}_{2} \mathrm{O}$ was observed on the green labelled geometry. 


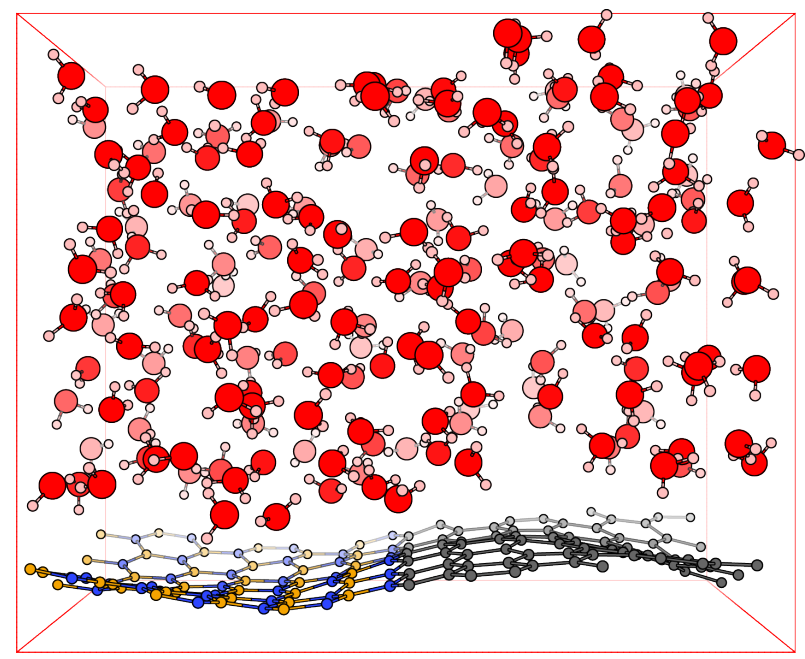

Figure 3. Ab Initio Molecular Dynamics Simulation Cell. Example of simulation cell with explicit water. The NinC junction geometry is represented along with $195 \mathrm{H}_{2} \mathrm{O}$. Hydrogen, boron, carbon, nitrogen and oxygen atoms are respectively represented in pink, orange, grey, blue and red. 

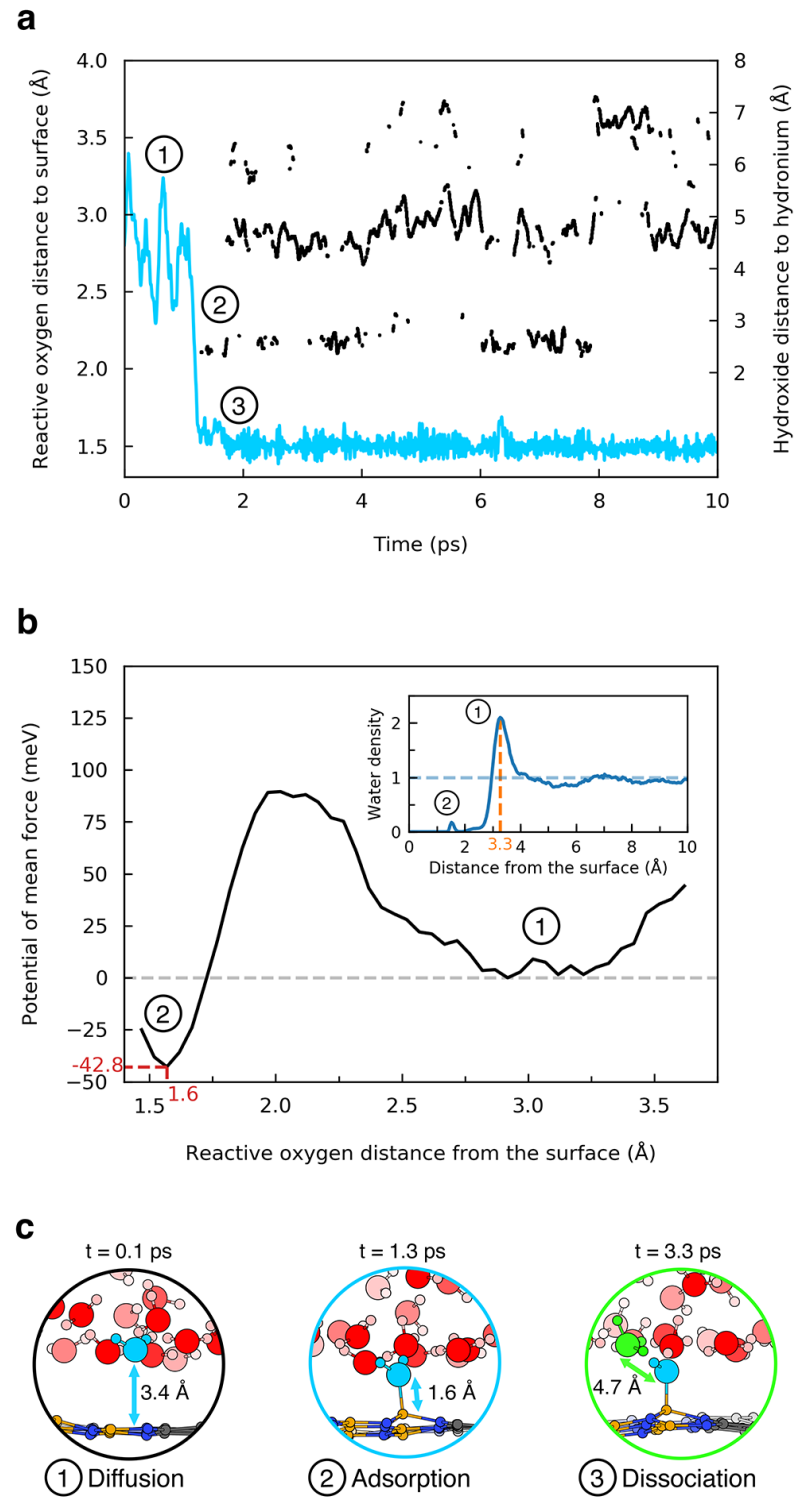

Figure 4. Spontaneous Dissociative Adsorption of Water. 10 ps long bias-free AIMD simulation of the NinC junction in water leading to the observation of a water dissociative adsorption. a) The distance to the surface of the oxygen adsorbing on a boron atom and the distance between the dissociated $\mathrm{OH}^{-}$and $\mathrm{H}_{3} \mathrm{O}^{+}$are respectively represented by a cyan line and black dots along the trajectory. b) Potential of mean force of a $\mathrm{H}_{2} \mathrm{O}$ with respect to its distance to the surface (black line), with the energy reference represented by a grey dotted horizontal line. The energy and position of the chemisorption well is indicated in red. (inset: The water density with respect to the distance of the surface with the horizontal and vertical lines respectively indicating the bulk density value and the position of the interfacial layer. The density corresponds to an average over the whole cell.) c) Snapshots of the dynamics with associated times and distances. The reactive $\mathrm{H}_{2} \mathrm{O}$ and $\mathrm{OH}^{-}$are both represented in cyan. Circled numbers correspond to the ones in $\mathbf{a}$ and $\mathbf{b}$. 